\title{
Detecting Node Misbehavior in Ad hoc Networks
}

\author{
M. Tamer Refaei ${ }^{\dagger}$, Yanxia Rong ${ }^{*}$, Luiz A. DaSilva ${ }^{\dagger}$, Hyeong-Ah Choi ${ }^{*}$ \\ $\uparrow$ Bradley Department of Electrical and Computer Engineering \\ Virginia Tech \\ Alexandria, VA 22314 \\ \{mtamer,ldasilva\}@vt.edu \\ *Department of Computer Science \\ George Washington University \\ Washington, DC 20052 \\ \{yxrong,hchoi\}@gwu.edu
}

Abstract

Ad hoc networks employ a decentralized unstructured networking model that relies on node cooperation for key network functionalities such as routing and medium access. In this paper, we develop a model based on the Sequential Probability Ratio Test to characterize how nodes can differentiate between routes that include misbehaving nodes (infected routes) and routes that do not. An advantage of the model is that the number of observations required to evaluate a route need not be determined in advance, which suits well the dynamic nature of ad hoc networks. We then outline a centralized and a localized approach to detect misbehaving nodes on infected routes identified by the model. Our evaluation shows that the localized approach is not only the better architectural choice for ad hoc networks but also results in a more accurate exposure of misbehaving nodes while incurring low false positives and low false negatives.

\section{Introduction}

Ad hoc networks typically consist of wireless batteryoperated nodes that dynamically form a communication network without the use of existing network infrastructure or centralized administration. This self-organizing networking model requires cooperation among nodes to support key network functionalities such as medium access and routing. In the routing functionality, nodes cooperate in route discovery to find operational routes to destinations outside of their transmission range, and participate in packet forwarding to deliver packets to these destinations. If nodes refuse to cooperate or tamper with the route discovery function, operational routes may not be found. If nodes refuse to cooperate with respect to the forwarding function, end-to-end packet delivery may not be possible. Such uncooperative behavior (which we characterize as misbehavior) can greatly degrade network performance and may even result in total communication breakdown. Consequently, it is important for both security and performance reasons to expose misbehaving nodes to limit the scope of their negative impact on network survivability.

Our objective in this paper is to accurately detect misbehavior with respect to the routing functionality in ad hoc networks. Based on the Sequential Probability Ratio Test (SPRT) [1] we develop a model to characterize how nodes differentiate between infected routes (routes that include misbehaving nodes) and clean routes (routes that do not include misbehaving nodes). A merit of SPRTs is that the number of observations required to test statistical hypotheses need not be determined in advance. This suits well the dynamic nature of ad hoc networks. Further, the number of observations used in SPRTs is greatly reduced (by $50 \%$ in most cases) when compared to other methods based on a fixed number of observations [1]. This allows for prompt decision making, which is a desirable characteristic in network security algorithms in order to limit the scope of damage to the network.

While the detection of infected routes only reduces the impact of misbehavior (by avoiding these routes, for example), it does not fully eliminate the impact of misbehavior (new infected routes may emerge). To eliminate the impact of misbehavior it is important to detect the misbehaving nodes on infected routes and react to such nodes (by blacklisting and eliminating them from all routes, for example). We outline two approaches, a centralized and a localized approach, for the detection of misbehaving nodes in infected routes identified by the SPRT model. The centralized approach assumes global knowledge of all infected routes in the network. The localized approach assumes only local knowledge of such routes. We evaluate the ability of each to detect misbehaving nodes and the false positives and false negatives each incurs. We conclude that the localized approach performs better than the centralized approach. A localized approach is also more appropriate due to the self-organizing, decentralized nature of ad hoc networks.

Our contribution in this work is twofold. First, we introduce a sequential analysis model for the detection of infected routes. The second contribution is the introduction and evaluation of two approaches, a centralized and a localized approach, for the detection of misbehaving nodes in infected routes identified by the model.

\subsection{Scope}

We assume that all nodes in the network are insider nodes, i.e. there is an authentication mechanism in place whereby a node can authenticate the source of packets it receives. Hence, nodes cannot forge packets. Each node has a unique identity and is not able to masquerade other nodes' identities or create new identities. We assume that links are bidirectional, which is a requirement of many existing MAC protocols such as the one adopted in IEEE 802.11 [2]. Nodes communicate over the wireless channel. We assume a communication environment where detection and isolation of misbehaving nodes is crucial to the survival of the network. Typically, this is the case in environments where the negative impact of node misbehavior on the network performance is much greater than the impact of fluctuations in channel conditions.

We classify node behavior with respect to the routing functionality at the network layer into cooperative or misbehaving. Cooperative nodes do not deliberately drop packets forwarded through them or tamper with routing information. Misbehaving nodes are of one of two types, selfish or malicious nodes. Selfishness is intentional passive misbehavior, where a node chooses not to fully participate in the packet forwarding functionality because it is concerned for its resources. Hence, a selfish node drops some or all packets forwarded through it. We stress that selfishness is passive behavior where nodes are motivated only by their self interest in conserving their resources. Hence, selfish nodes do not collude with each other or exert additional effort to camouflage their 
behavior such as slander attacks. Maliciousness, on the other hand, is intentional active misbehavior, where a node's aim is to deliberately disrupt the network. A malicious node is capable of launching blackhole or greyhole attacks [3]. Other classes of outsider attacks or insider node misbehavior at layers other than the network layer are outside the scope of this paper.

The impact of malicious node misbehavior is usually greater than that of selfish node misbehavior. We argue that a mechanism that is capable of identifying passive selfish node misbehavior should be able to identify certain types of malicious node misbehavior faster, due to the more aggressive nature of the latter. We focus on selfish misbehavior, primarily considering nodes that misbehave at the network layer by dropping some or all packets forwarded through them. Much of the analysis and design described here, however, also applies to certain types of blackhole and greyhole malicious attacks.

This paper is organized as follows. In section 2 we introduce the network model. In section 3 we introduce the sequential probability ratio test for detection of infected routes. In section 4 we evaluate the model accuracy. We introduce and evaluate two approaches for the detection of misbehaving nodes in section 5. In section 6 , we discuss related work.

\section{Network Model}

Consider an ad hoc network with nodes $\mathrm{N}=\{1,2, \ldots, n\}$ where nodes are either selfish or cooperative. We assume the network has selfish nodes in $\mathrm{M} \subset \mathrm{N}$. Hence, $|\mathrm{N}|$ is the number of nodes in the network, $|\mathrm{M}|$ is the number of selfish nodes, and $|\mathrm{N}|-|\mathrm{M}|$ is the number of cooperative nodes.

Packet drops occur due to two classes of factors: node misbehavior (one or more nodes along a route act selfishly, dropping packets); and network environment factors (under which we include congestion at the network layer, contention at the data link layer, physical communication impairments such as fading, etc.). Let $P_{\text {drop }}$ represent the probability that a data packet will be dropped due to network environment factors. For simplicity of presentation, we assume that all nodes $i \in \mathrm{N}$ will experience the same dropping probability due to environmental factors. The extension to the model to consider different drop probabilities at different nodes is straight-forward. As for packet drops due to misbehavior in the network, we assume that each selfish node $i \in \mathrm{M}$ will drop a data packet forwarded through it with probability $P_{\text {selfish }}$. We assume a communication environment where detection and isolation of misbehaving nodes is crucial to the survival of the network. Typically, this is the case when the negative impact of node selfishness (modeled as $\left.P_{\text {selfish }}\right)$ on network performance is much greater than deteriorating channel and/or network conditions (modeled as $\left.P_{\text {drop }}\right)$.

Consider, for now, symmetric routes in the network (we recognize the existence of asymmetric routes; as we will see later, as long as a reasonable proportion of all routes is symmetric, it is possible to accurately detect infected routes and misbehaving nodes). Define $R^{s \leftrightarrow d}=\left\{h_{0}, \ldots, h_{k}\right\}$ as an ordered set of nodes representing the route from source node $s=h_{0}$ up to the last node $h_{k}$ before the destination node $d(k+1$ is the number of hops on the route). Let the ordering relationship on $R^{s \leftrightarrow d} \quad$ be $\quad \forall x, y \in R^{s \leftrightarrow d}, x<y \Leftrightarrow h o p(x, s \leftrightarrow d)<h o p(y, s \leftrightarrow d)$, where $h o p(x, s \leftrightarrow d)$ is the number of hops between source node $s$ and node $x$ on the route from source node $s$ to destination node $d$. Let $R_{i}^{s \leftrightarrow d} \subset R^{s \leftrightarrow d}$ be the portion of the route $R^{s \leftrightarrow d}$ between source node $s$ and destination node $d$ that starts at node $h_{i}$ and ends at node $h_{k}$. Hence, $R_{i}^{s \leftrightarrow d}=\left\{h_{i}, \ldots, h_{k}\right\} \quad 0 \leq i \leq k \quad(k-i+1$ is the number of hops on $R_{i}^{s \leftrightarrow d}$ ). A route $R_{i}^{s \leftrightarrow d}$ is considered "infected" if $\exists h_{j} \in R_{i}^{s \leftrightarrow d}$ such that $h_{j} \in \mathrm{M}, i<j \leq k$; otherwise it is "clean."

We assume some mechanism is available to provide feedback on end to end packet delivery. If a reliable transport layer protocol such as TCP is adopted, this feedback is provided at the transport layer in the form of TCP acknowledgements. Otherwise, application layer acknowledgements could be used.

\section{Detection of Infected Routes}

\subsection{Overview of Sequential Probability Ratio Test (SPRT)}

Consider two hypotheses $H_{1}$ and $H_{0}$, where either $H_{1}$ or $H_{0}$ is true but not both, and two corresponding conditional probabilities, $P\left[x \mid H_{1}\right]$ and $P\left[x \mid H_{0}\right]$. To make a decision whether $H_{1}$ or $H_{0}$ is true we make a sequence of observations $x_{1}, x_{2}, \ldots$. For each observation $x_{n}, n \geq 1$, if $P\left[x_{n} \mid H_{0}\right] \neq 0$ we calculate the ratio $\frac{P\left[x_{n} \mid H_{1}\right]}{P\left[x_{n} \mid H_{0}\right]}$ and accumulate the value $T_{n}=T_{n-1} * \frac{P\left[x_{n} \mid H_{1}\right]}{P\left[x_{n} \mid H_{0}\right]}$, where $T_{0}=1$. We then examine the value of $T_{n}$. If $T_{n}$ is large, it implies that the sequence of observations $x_{1}, \ldots, x_{n}$ made so far are more likely to have been generated under $H_{1}$ than under $H_{0}$. If $T_{n}$ is small, the converse is true. If $T_{n}$ is not sufficiently small or large to choose between $H_{1}$ and $H_{0}$ we make another observation $x_{n+1}$.

In making a decision between $H_{1}$ and $H_{0}$, it is possible to erroneously decide that $H_{1}$ is true while in reality $H_{0}$ is true (a false positive), or that $H_{0}$ is true while in reality $H_{1}$ is true (a false negative). To limit the probability of false positives by $\alpha$ and that of false negatives by $\beta$, we select two threshold values $A$ and $B$, with $B<A$. After making a sequence of observations $x_{1}, \ldots, x_{n}$ a decision is made that $H_{1}$ is true if $T_{n} \geq A$, or that $H_{0}$ is true if $T_{n} \leq B$ and the test terminates. We make an additional observation $x_{n+1}$ if $B<T_{n}<A$. It was shown in [1] that the values of $A$ and $B$ are bounded by $A \leq \frac{1-\beta}{\alpha}$ and $B \geq \frac{\beta}{1-\alpha}$ and that by using values of $A=\frac{1-\beta}{\alpha}$ and $B=\frac{\beta}{1-\alpha}$ the test provides adequate level of precision. 


\subsection{Sequential Probability Ratio Test for Detection of Infected Routes}

In this section we define our model for detection of infected routes. The mathematical formulation of the model considers routes to be symmetric. While we recognize the existence of asymmetric routes, we will later see that as long as a reasonable proportion of routes are symmetric our model works well, achieving high accuracy in detection of infected routes. We also note that ad hoc routing protocols typically attempt to construct routes that are symmetric [4] [5] [6].

Let $H_{1}$ be the hypothesis that a given route is infected and $H_{0}$ be the hypothesis that the route is clean. We need to identify which hypothesis holds. As mentioned earlier, we assume some mechanism is available to provide feedback on end to end packet delivery. If TCP is adopted, we use packet ACKnowledgements as observations of successful packetdelivery events and packet RETransmissions as observations of failed packet-delivery events. Let $T\left(i, R_{i}^{s \leftrightarrow d}\right)$ be an evaluation of route $R_{i}^{s \leftrightarrow d}$ by node $h_{i} \in R^{s \leftrightarrow d}$. Upon encountering an ACK or a RET observation $x_{n}$ at node $h_{i}$ about route $R_{i}^{s \leftrightarrow d}$, the node evaluates

$$
T\left(i, R_{i}^{s \leftrightarrow d}\right)_{n}=\left\{\begin{array}{l}
T\left(i, R_{i}^{s \leftrightarrow d}\right)_{n-1} * \frac{P\left[A C K \mid H_{1}\right]}{P\left[A C K \mid H_{0}\right]} \text { if } x_{n}==A C K \\
T\left(i, R_{i}^{s \leftrightarrow d}\right)_{n-1} * \frac{P\left[R E T \mid H_{1}\right]}{P\left[R E T \mid H_{0}\right]} \text { if } x_{n}==R E T
\end{array}\right\}
$$

for $n \geq 1$, with $T\left(i, R_{i}^{s \leftrightarrow d}\right)_{0}=1 . P[A C K]$ and $P[R E T]$ are the probabilities of observing an ACK or a RET on $R_{i}^{s \leftrightarrow d}$ at $h_{i}$, respectively. To determine whether the route $R_{i}^{s \leftrightarrow d}$ is infected or clean, node $h_{i} \in R^{s \leftrightarrow d}$ performs the following test: if $T\left(i, R_{i}^{s \leftrightarrow d}\right)_{n} \geq A$ a conclusion is made that $H_{1}$ is true, if $T\left(i, R_{i}^{s \leftrightarrow d}\right)_{n} \leq B$ a conclusion is made that $H_{0}$ is clean. Note that some asymmetric routes may observe only RETs or only ACKs, which indicates that it is possible to erroneously classify a clean route as infected, resulting in a false negative, or an infected route as clean, resulting in a false positive. We will see in section 4.2 that as long as a reasonable proportion of routes are symmetric the impact of such cases on the system's accuracy is insignificant. In the next section we will determine how $T\left(i, R_{i}^{s \leftrightarrow d}\right)$ is evaluated.

\subsection{Evaluating $T\left(i, R_{i}^{s \leftrightarrow d}\right)$}

For each instance of a data packet transmitted by a source node of a flow, our model considers $P[A C K]+P[R E T]=1$ at any intermediate node on a route, and that ACK and RET events are mutually exclusive for this packet instance. In other words, the only two possible fates of an instance of a data packet transmitted by the source node are: successful delivery to the destination, and hence an ACK is generated by the destination in response, or packet loss and a RET is generated by the source. Hence, at any node $h_{i}$ on a route $R^{s \leftrightarrow d}$ either an ACK or a RET should be observed for each instance of a data packet sent by node $S$, but not both. This is supported by the following assumptions:

- Any observation $x_{n}==A C K$ witnessed by a node $h_{i}$ on $R^{s \leftrightarrow d}$ will also be witnessed by all nodes $h_{j}$ where $k \geq j>i$. By the same token, any observation $x_{n}==R E T$ witnessed by a node $h_{i}$ on $R^{s \leftrightarrow d}$ will also be witnessed by all nodes $h_{j}$ where $0 \leq j<i$.

- Each node $h_{i}$ on a route $R^{s \leftrightarrow d}$ performs the test $T\left(i, R_{i}^{s \leftrightarrow d}\right)$ to evaluate $R_{i}^{s \leftrightarrow d}$ only, and not the complete route $R^{s \leftrightarrow d}$. Hence any RET event for an instance of a data packet where an ACK has already been observed at node $h_{i}$ (i.e. the ACK might have been dropped on $R^{s \leftrightarrow d} \backslash R_{i}^{s \leftrightarrow d}$ ) is treated by node $h_{i}$ as a new data packet.

Accordingly, we can say that $P\left[A C K \mid H_{1}\right]+P\left[R E T \mid H_{1}\right]=1$ and that $P\left[A C K \mid H_{0}\right]+P\left[R E T \mid H_{0}\right]=1$.

Consider an ACK or RET observation made by node $h_{i} \in R^{s \leftrightarrow d}$ about a data packet sent on $R_{i}^{s \leftrightarrow d} . \quad P[A C K]=$ $P[$ Data_Delivered $] * P[A C K$ _observed $\mid$ Data_Delivered $]$, where $P[$ Data_Delivered $]$ indicates the probability that the data packet was delivered at the destination. This means that the data packet was not dropped by any node on the route $R_{i}^{s \leftrightarrow d}$ due to network environment factors or as a result of selfish misbehavior. On the other hand, $P\left[A C K \_\right.$Observed $\mid$Data_Delivered $]$indicates the probability that the ACK corresponding to a successfully delivered data packet was observed at node $h_{i}$, which means that it was not dropped by any node on the route $R_{i}^{s \leftrightarrow d}$ due to network environment factors or as a result of selfish misbehavior.

The value of $P[$ Data_Delivered $]$ as well as the value of $P[$ ACK_Observed $\mid$ Data_Delivered $]$ for a node $h_{i} \in R^{s \leftrightarrow d}$ are affected by the number of selfish nodes on the route $R_{i}^{s \leftrightarrow d}$. The higher the number of selfish nodes on the route the lower the values of $P[$ Data_Delivered $]$ and $P\left[A C K_{\text {_Observed }} \mid\right.$ Data_Delivered $]$. Let $a=\left|R_{i}^{s \leftrightarrow d} \cap \mathrm{M}\right|$ be the actual number of selfish nodes on the route $R_{i}^{s \leftrightarrow d}$, noting that $a=0$ if the route is clean and $1 \leq a \leq \min (k-i,|\mathrm{M}|)$ otherwise. Let $P_{\Pi(\text { selfish })}=1-\left(1-P_{\text {selfish }}\right)^{a}$ be the aggregate impact of selfishness on infected route $R_{i}^{s \leftrightarrow d}$. Accordingly, $P[$ Data_Delivered $]=\left(1-P_{d r o p}\right)^{k-i} *\left(1-P_{\Pi(\text { selfish })}\right) \quad$ and $P[$ ACK_Observed $\mid$ Data_Delivered $]=\left(1-P_{\text {drop }}\right)^{k-i+1} *$ $\left(1-P_{\Pi(\text { selfish })}\right)$. We can then calculate $P[A C K]=\left(1-P_{d r o p}\right)^{2(k-i)+1} *\left(1-P_{\Pi(\text { selfish })}\right)^{2}$. Hence, we can find the values of $P\left[A C K \mid H_{0}\right], P\left[A C K \mid H_{1}\right], P\left[R E T \mid H_{0}\right]$, and $P\left[R E T \mid H_{1}\right]$ as follows: 
- If $R_{i}^{s \leftrightarrow d}$ is clean then $P\left[A C K \mid H_{0}\right]=\left(1-P_{d r o p}\right)^{2(k-i)+1}$ and $P\left[R E T \mid H_{0}\right]=1-\left(1-P_{\text {drop }}\right)^{2(k-i)+1}$.

$$
\begin{aligned}
& \text { - If } \quad R_{i}^{s \leftrightarrow d} \quad \text { is } \quad \text { infected } \\
& P\left[A C K \mid H_{1}\right]=\left(1-P_{d r o p}\right)^{2(k-i)+1}\left(1-P_{\Pi(\text { selfish })}\right)^{2} \\
& P\left[R E T \mid H_{1}\right]=1-\left[\left(1-P_{\text {drop }}\right)^{2(k-i)+1}\left(1-P_{\Pi(\text { selfish })}\right)^{2}\right] .
\end{aligned}
$$

then and

Hence, given observation $x_{n}$ at node $h_{i}$ about route $R_{i}^{s \leftrightarrow d}$ :

- If $x_{n}==A C K: T\left(i, R_{i}^{s \leftrightarrow d}\right)_{n}=T\left(i, R_{i}^{s \leftrightarrow d}\right)_{n-1}\left(1-P_{\Pi(\text { selfish })}\right)^{2}$

- If $x_{n}==R E T: T\left(i, R_{i}^{s \leftrightarrow d}\right)_{n}=T\left(i, R_{i}^{s \leftrightarrow d}\right)_{n-1} *$

$$
\frac{1-\left[\left(1-P_{d r o p}\right)^{2(k-i)+1}\left(1-P_{\Pi(\text { selfish })}\right)^{2}\right]}{1-\left(1-P_{d r o p}\right)^{2(k-i)+1}}
$$

\section{Performance Analysis}

We now evaluate the model discussed in the previous section to assess its ability to accurately distinguish between infected and clean routes. We realize an implementation of the model that does not require perfect knowledge of $P_{d r o p}$, $P_{\Pi \text { (selfish) }}$, or the exact number of hops on a route and implement it using ns-2 to evaluate its accuracy.

\subsection{Model Implementation}

We realize an implementation of the model that estimates model parameters as follows:

- $P_{\text {drop }}:$ Each node monitors the network conditions surrounding it for packet drop and packet forward events and estimates the value of $P_{d r o p}$ accordingly.

- $P_{\Pi(\text { selfish })}$ : The value of $P_{\Pi(\text { selfish })}$ is not known because the exact number of misbehaving nodes (i.e. the value of $a$ in the model) on a route is not known. However, we assume that $P_{\Pi(\text { selfish })}$ is a network parameter that is set according to the network objective. Let $\widetilde{P}_{\Pi(\text { selfish })}$ be the value of this network parameter and let $\bar{P}_{\Pi(\text { selfish })}$ be the actual value of $P_{\Pi(\text { selfish })}$ on a route. $\widetilde{P}_{\Pi \text { (selfish) }}$ is set according to the aggregate level of selfishness on a route that the network can tolerate. For example, if the value of $\widetilde{P}_{\Pi(\text { selfish })}$ is set to $\widetilde{P}_{\Pi(\text { selfish })}=50 \%$ then the objective of the network is to detect infected routes where the aggregate selfishness on the route $\bar{P}_{\Pi(\text { selfish })} \geq 50 \%$. This also implies that the network can tolerate infected routes where the aggregate selfishness $\bar{P}_{\Pi(\text { selfish })}<50 \%$.

- The number of hops from a node to a route's destination is available to the node in its routing table. The number of hops from a route's destination to a node may not be known with certainty. A first order approximation is to assume that the total number of hops on the route $=2 *$ the number of hops on the forward route.

- We consider that some routes may be constructed asymmetrically by the routing protocol.
An evaluation record $T\left(i, R_{i}^{s \leftrightarrow d}\right)$ is maintained for each active route ${ }^{1} R_{i}^{s \leftrightarrow d}$ at a node $h_{i} \in R^{s \leftrightarrow d}$. The record is initialized and updated at node $h_{i}$ for each observation $x_{n}$ about $R_{i}^{s \leftrightarrow d}$ as discussed in section 3.2. Whenever $R_{i}^{s \leftrightarrow d}$ is updated in the routing table of node $h_{i}$ the value of $T\left(i, R_{i}^{s \leftrightarrow d}\right)$ is reset to its initial value. Hence, each instance of a route is evaluated independently. The model's $A$ and $B$ thresholds are maintained at each node and a decision whether a route is infected or clean is performed as shown in section 3.2.

\section{Table 1 Model Parameters}

\begin{tabular}{c|c|c|c}
$\alpha$ & $\beta$ & $A$ & $B$ \\
\hline $10 \%$ & $10 \%$ & 9 & 0.11
\end{tabular}

\subsection{Evaluation}

In this section we evaluate our model implementation. Our goal is to assess the ability of the model implementation to accurately distinguish between infected and clean routes given its estimation of the model's parameters. Since $P_{\Pi(\text { selfish })}$ is an important model parameter whose value is set according to the network objective, we also evaluate how the increase in $\bar{P}_{\Pi(\text { selfish })}$, given a particular value of $\widetilde{P}_{\Pi(\text { selfish })}$, impacts the accuracy of distinguishing between infected and clean routes. Accordingly, we use two sets of simulation settings:

- Setting S1: $\bar{P}_{\Pi(\text { selfish })}=75 \%$ and $\widetilde{P}_{\Pi(\text { selfish })}=50 \%$.

- Setting S2: $\bar{P}_{\Pi(\text { selfish })}=100 \%$ and $\widetilde{P}_{\Pi(\text { selfish })}=50 \%$.

For both settings we adopt the model parameter values shown in Table 1. For each setting we run $250 \mathrm{~ns}-2$ simulations using 5 different TCP traffic loads (each traffic load is run 50 times with flow source and destination pairs selected at random for each run). Traffic is generated for each flow using a CBR (Constant Bit Rate) traffic generator that generates approximately 300 data packets per flow. The network considered is composed of 64 nodes arranged in an $8 \mathrm{X} 8$ grid. The communication range is set such that each node has 4 neighbors, with the exception of edge nodes, which have 3 neighbors, and corner nodes, which have 2 neighbors. The network adopts IEEE 802.11 [2] for medium access and AODV [4] for routing. We use false positives and false negatives as defined in [1] as the basis for accuracy evaluation. We observe the decisions made by each node (i.e. $H_{1}$ or $H_{0}$ ) and examine the false positives and false negative that result from all decisions made by all nodes.

We compare the false positives and false negatives resulting from each setting of the model implementation to the theoretical bounds of $\alpha$ and $\beta$. Figure 1 shows that false positives in both settings are bounded by $\alpha$ under all traffic loads. It also shows that false positives are almost identical for both settings. False positives occur when a clean route is identified as infected due to retransmissions on the route. Since the route is actually clean, the value of $\bar{P}_{\Pi \text { (selfish) }}$ does not impact retransmissions. Hence, false positives are likely to be

\footnotetext{
${ }^{1}$ A route is active if its routing table entry has not expired.
} 


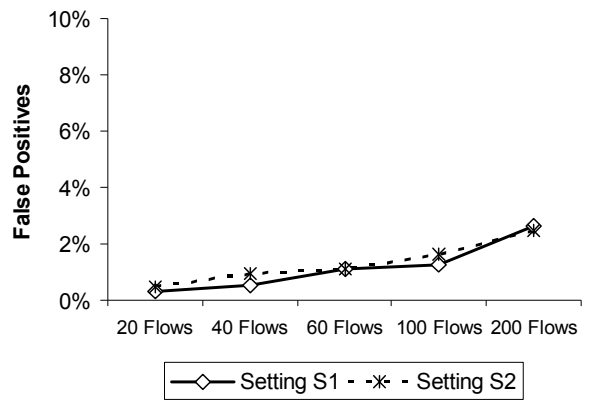

Figure 1 Model Accuracy: False Positives

the same for a given value of $\widetilde{P}_{\Pi(s e l f i s h)}$ irrespective of the value of $\bar{P}_{\Pi(\text { selfish })}$. With respect to false negatives, Figure 2 shows that settings $S 1$ and $S 2$ are bounded by $\beta$. Figure 2 shows that false negatives decrease as the value of $\bar{P}_{\Pi(\text { selfish })}$ increases. A false negative occurs when an infected route is falsely identified as clean because of ACK events observed on the route. As $\bar{P}_{\Pi(s e l f i s h)}$ increases the likelihood that an ACK event is observed on an infected route decreases. Hence, false negatives decrease.

Simulation results show that even though the model parameters are estimated with some error, false negatives and false positives remain bounded by $\alpha$ and $\beta$ respectively. We noticed that given a value of $\widetilde{P}_{\Pi(\text { selfish })}$ that the system can tolerate, system accuracy increases as $\bar{P}_{\Pi(\text { selfish })}-\widetilde{P}_{\Pi(\text { selfish })}$ increases. In our simulations, we considered that some routes may be asymmetric. An assessment of the proportion of asymmetric routes in the simulations revealed that between $10 \%-20 \%$ of all routes were constructed asymmetrically by the routing protocol. The accuracy achieved by the model suggests that as long as the proportion of symmetric routes is reasonably high, asymmetric routes have insignificant impact on the model's accuracy. Since routing protocols typically attempt to construct routes symmetrically [4] [5] [6], it is reasonable to assume that a large proportion of routes will be symmetric.

\section{Detecting Selfish Nodes from Infected Routes}

We now consider identifying selfish nodes from the routes reported as infected by the model. While the detection of infected routes reduces the impact of misbehavior (by avoiding these routes, for example), it does not fully eliminate the impact of misbehavior (new infected routes may emerge). To eliminate the impact of misbehavior it is important to detect and react to misbehaving nodes by isolating them from the network, for example. We propose two approaches to detect selfish nodes on infected routes: a centralized approach and a localized approach. The centralized approach assumes knowledge of all infected routes in the network, which is the case if a central authority exists to which nodes report routes they identify as infected, or if nodes exchange such information with one another. The localized approach assumes that each node knows only about routes it identified as infected and only knows about its next hop neighbors on these routes. To assess the effectiveness of each approach we use three metrics: exposure (proportion of selfish

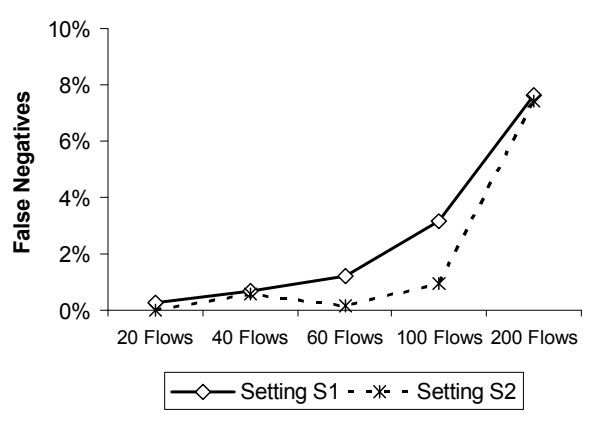

Figure 2 Model Accuracy: False Negatives

nodes identified as such), false positives (proportion of cooperative nodes falsely identified as selfish), and false negatives (proportion of selfish nodes falsely identified as cooperative). In defining these metrics, we use the indicator function $1_{\{\mathrm{A}\}}=\left\{\begin{array}{l}1, \text { if } A \text { is true } \\ 0, \text { if } A \text { is false }\end{array}\right.$.

\subsection{Centralized Approach}

This approach assumes knowledge of all routes identified as infected by all nodes in the network. We construct a sorted list of nodes that occur most frequently in infected routes. Nodes positioned high in the list are identified as selfish. This approach works as follows:

- Define $R_{\text {infected }}=\bigcup_{i \in \mathrm{N}} R_{\mathrm{infected}}^{\mathrm{i}}$ as the set of all routes identified as infected, where $R_{\text {infected }}^{\mathrm{i}}$ is the set of infected routes identified by node $i, \forall i \in \mathrm{N} \backslash \mathrm{M}$. Let $R I=\left|R_{\text {infected }}\right|$.

- Let $G(i)=\sum_{r \in R_{\text {infected }}} 1_{i \in r}$ be the ranking of node $i, \forall i \in \mathrm{N}$ based on the frequency of its appearance in infected routes. For example, $G(i)=2$ if node $i$ appears in two routes in $R_{\text {infected }}$.

- Using $G$, we construct $M F N$, the list of most frequent nodes in $R_{\text {infected }}$. Most if not all selfish nodes should be in this list.

Figures 3,4 , and 5 show a scatter of the average ranking of the nodes in $M F N$ under different traffic loads for $P_{\Pi(\text { selfish })}=100 \%\left(P_{\Pi(\text { selfish })}=75 \%, 50 \%\right.$ show similar results $)$. The set of $R_{\text {infected }}$ was constructed out of 50 simulation runs. The figures show that it is difficult to draw a clear line between the rankings of selfish and cooperative nodes. Figure 9 further illustrates this observation by comparing the average ranking of selfish and cooperative nodes indicating that the two are very close in value. Given $T H$, the threshold ranking between selfish and cooperative nodes, we define the following:

- Exposure: $\frac{\sum_{i \in \mathrm{MFN}} 1_{G(i) \geq T H \wedge i \in \mathrm{M}}}{|\mathrm{M}|}$

- False Positives: $\frac{\sum_{i \in \mathrm{MFN}} 1_{G(i) \geq T H \wedge i \in \mathrm{N} \backslash \mathrm{M}}}{|\mathrm{N} \backslash \mathrm{M}|}$

- False Negatives: $\frac{\sum_{i \in \mathrm{MFN}} 1_{G(i)<T H \wedge i \in \mathrm{M}}}{|\mathrm{M}|}$ 


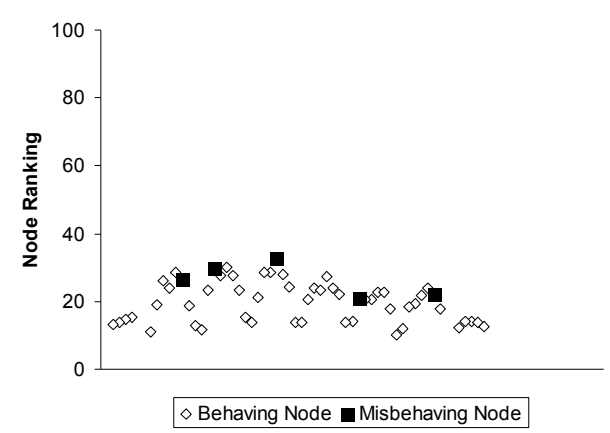

Figure 3 Ranking of MFN - 60 flows -

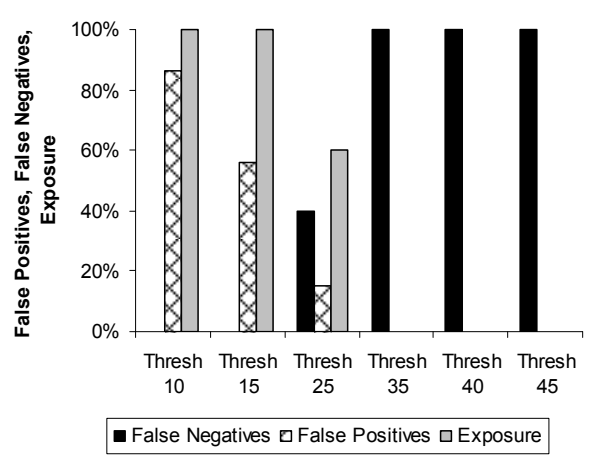

Figure 6 False Positives, False Negatives, and Exposure for 60 flows

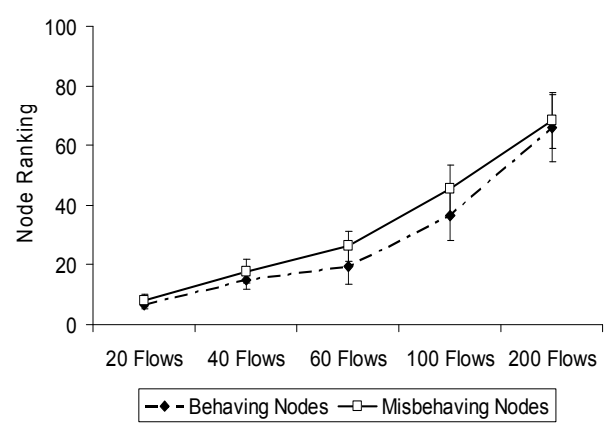

Figure 9 Average and standard deviation of rankings of behaving and misbehaving nodes (centralized approach)

Figures 6, 7, and 8 show the values of exposure, false positives and false negatives for six different values of $T H$ under different traffic loads. Note that a good mechanism for identifying misbehaving nodes should achieve high exposure, low false positives, and low false negatives. From the figures, it is clear that it is not possible to get both high exposure and low false positives using the centralized approach. To achieve 100\% exposure, false positives was at a minimum of $55.93 \%$ at 60 flows, $47.46 \%$ at 100 flows, and $79.66 \%$ at 200 flows. This is because the rankings of selfish and cooperative nodes are within close range, which makes it hard to identify selfish nodes with reasonable accuracy.

\subsection{Localized Approach}

The localized approach assumes that each node $i, \forall i \in \mathrm{N} \backslash \mathrm{M}$ has a set of routes $R_{\text {infected }}^{\mathrm{i}}$ that it identified as

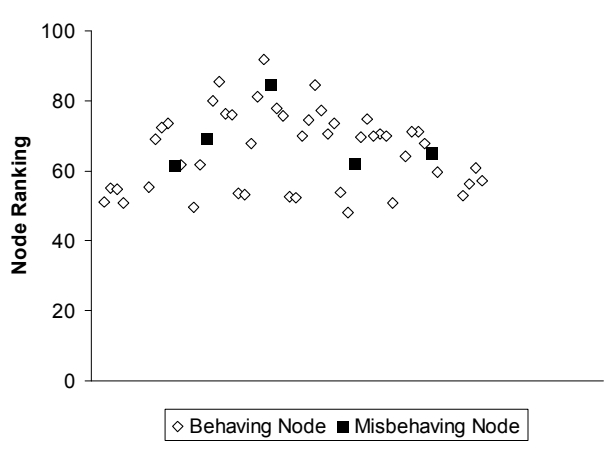

Figure 5 Ranking of MFN - 200 flows -

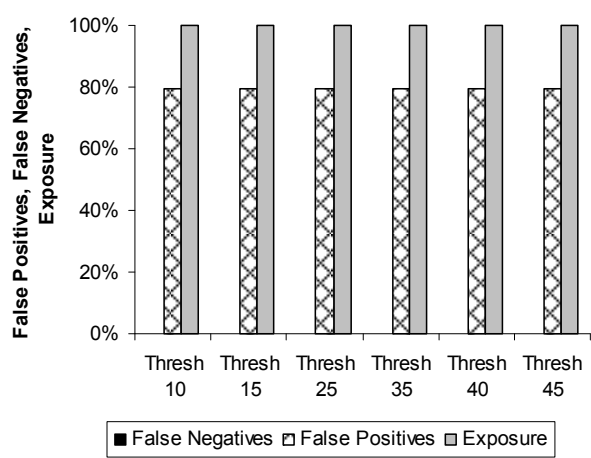

Figure 8 False Positives, False Negatives, and Exposure for 200 flows

infected and that it knows only its next hop neighbor on each of these routes. Let $d(i, j)$ be the distance in hops between nodes $i$ and $j, \forall i, j \in \mathrm{N}$. Each node ranks its next hop neighbors on these routes as follows:

- Let $R_{\text {infected }}^{\mathrm{i}}$ be the set of next hop neighbors on infected routes identified by a node $i, \forall i \in \mathrm{N} \backslash \mathrm{M}$.

- Define $G_{i}(j)=\sum_{r \in R_{\text {inf ected }}^{i}} 1_{j \in r}$, the ranking assigned by node $i$ to node $j$ based on the frequency its appearance as a next hop to node $i$ in $R_{\text {infected }}^{\mathrm{i}}, \forall i \in \mathrm{N} \backslash \mathrm{M}, j \in \mathrm{N}$ where $d(i, j)=1$.

Figures 10, 11, and 12 show a scatter of the rankings of nodes by their neighbors averaged over 50 simulation runs under different traffic loads for $P_{\Pi(\text { selfish })}=100 \%\left(P_{\Pi(\text { selfish })}=75 \%, 50 \%\right.$ show similar results). The figures show that the rankings of selfish nodes are higher than those of cooperative nodes and the difference between both becomes clearer as the traffic load increases. Figure 16 further illustrates this observation by plotting the average ranking of a selfish node versus that of a cooperative node. Using six different values of $T H$ (threshold ranking between selfish and cooperative nodes), we evaluate the localized approach under different traffic loads by measuring its exposure, false positives, and false negatives defined as follows:

- Exposure: $\frac{\sum_{i \in \mathrm{N} \backslash \mathrm{M}} \sum_{j \in \mathrm{M}} 1_{d(i, j)=1 \wedge G_{i}(j) \geq T H}}{\sum_{i \in \mathrm{N} \backslash \mathrm{M}} \sum_{j \in \mathrm{M}} 1_{d(i, j)=1}}$ 


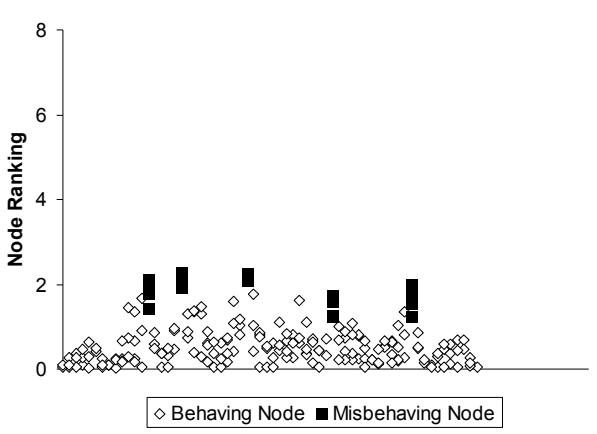

Figure 10 Ranking of nodes for their neighbors - 60 flows -

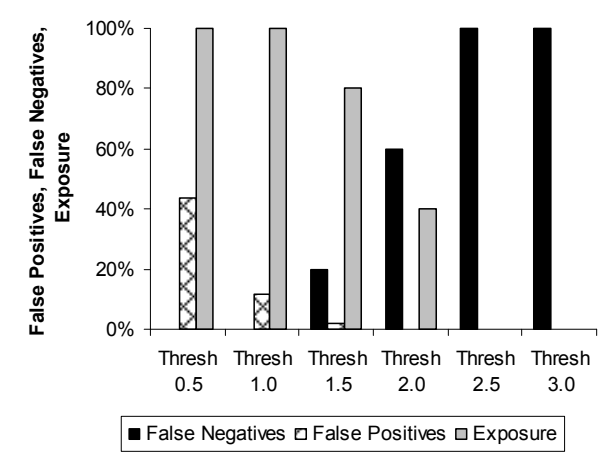

Figure 13 False Positives, False Negatives, and Exposure for 60 Flows

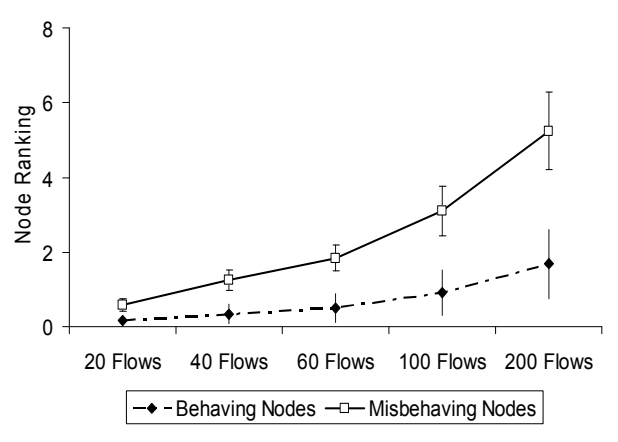

Figure 16 Average and standard deviation of rankings of behaving and misbehaving nodes (localized approach)

- False Positives: $\frac{\sum_{i \in \mathrm{N} \backslash \mathrm{M}} \sum_{j \in \mathrm{N} \backslash \mathrm{M}} 1_{d(i, j)=1 \wedge G_{i}(j) \geq T H}}{\sum_{i \in \mathrm{N} \backslash \mathrm{M}} \sum_{j \in \mathrm{N} \backslash \mathrm{M}} 1_{d(i, j)=1}}$

- False Negatives: $\frac{\sum_{i \in \mathrm{N} \backslash \mathrm{M}} \sum_{j \in \mathrm{M}} 1_{d(i, j)=1 \wedge G_{i}(j)<T H}}{\sum_{i \in \mathrm{N} \backslash \mathrm{M}} \sum_{j \in \mathrm{M}} 1_{d(i, j)=1}}$

Figures 13, 14, and 15 show that high exposure and low false positives can be achieved in the localized approach. To achieve $100 \%$ exposure, false positives was at a minimum of $11.48 \%$ at 60 flows, $14.97 \%$ at 100 flows, and $9.09 \%$ at 200 flows. The choice of $T H$ plays an important role in determining the value of exposure and false positives. To maintain false positives below $10 \%$ for example, a threshold value of 1.5 for 60 flows should be used, 2.0 for 100 flows, and 3.0 for 200 flows.

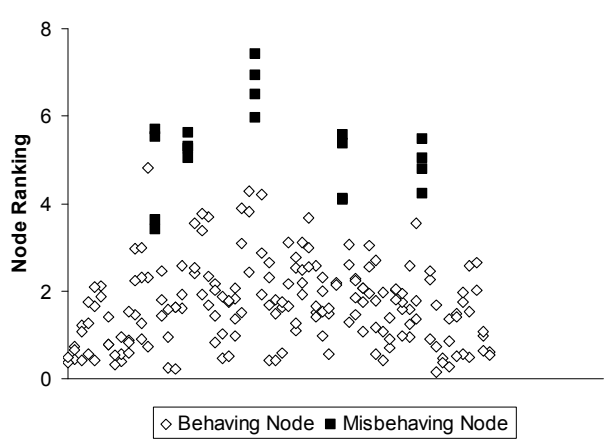

Figure 12 Ranking of nodes for their neighbors - 200 flows -

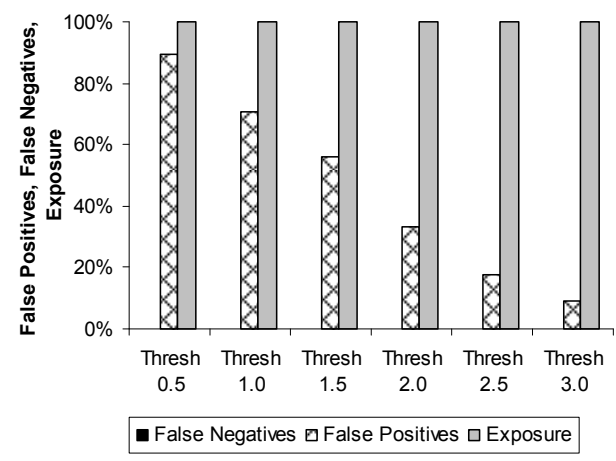

Figure 15 False Positives, False Negatives, and Exposure for 200 Flows

This corresponds to an exposure of $80 \%$ at 60 flows, $95 \%$ at 100 flows, and $100 \%$ at 200 flows. Accordingly, the value of $T H$ can be a system parameter that is adapted according to the traffic load a node is experiencing.

\section{Related Work}

Previous work highlighted the importance of securing ad hoc networks [7] [8] [9]. A threat model described in [10] distinguishes between selfish and malicious nodes. Selfishness is intentional passive misbehavior and maliciousness is intentional active misbehavior. To address the problem of node misbehavior in ad hoc networks three classes of solutions have been proposed: secure routing protocols, cooperation incentives, and node behavior evaluation. The first class ([3] [11] [12]) aims to secure the establishment and maintenance of routes in routing protocols such as AODV [4] and DSR [5]. This can protect the network against malicious misbehavior with respect to route discovery. However, it does not protect the network against selfish misbehavior. The second class ([13] [14]) aims to provide incentives for nodes to cooperate in such a way that rational nodes lose if they do not cooperate. Incentives are usually in the form of a payment that a node needs to make for services it requests from other nodes and credit that a node gets for services it provides for others. With respect to packet forwarding, nodes need to have enough credit to be able to send their packets through the network, which they gain by forwarding packets for others. This class of solutions requires overhead for accounting of credit and payments and is not effective against malicious misbehavior. The third class of 
solutions ([15] [16] [17] [16] [18] [19] [20] [21] [22] [23] [24] [25]) are reputation management systems that evaluate node behavior and detect misbehaving nodes accordingly. Their goal is to: encourage nodes to cooperate, evaluate nodes behavior based on their cooperation level, distinguish between cooperative and misbehaving nodes, and appropriately react to misbehaving nodes. This class of solutions is effective against both selfish and malicious misbehavior.

An approach that combines the second the third class of solutions is introduced in [26]. Nodes monitor and evaluate their neighbors' behavior. Through a localized collaborative approach, credit is issued to nodes whose neighbors agree are cooperative. Once misbehaving nodes are detected by the majority of their neighbors, they are issued no credit and hence isolated from the network. In [27], a sequential probability ratio test based algorithm was introduced to detect uncooperative behavior at the MAC layer in ad hoc networks. In [28] the problem of misbehavior at the MAC layer is introduced and formulated as a minmax robust sequential detection problem.

\section{Conclusion}

In this paper we introduced a Sequential Probability Ratio Test (SPRT) to distinguish between infected and clean routes. SPRTS operate faster compared to other test procedures and do not require a predefined number of observations. We evaluated the model accuracy using ns- 2 simulations. Based on the model, we developed two approaches for detecting misbehaving nodes, a centralized and a localized approach. We evaluated each and showed that the localized approach performs much better, achieving high exposure and low false positives.

\section{References}

[1] A. Wald, "Sequential Analysis, J. Wiley \& Sons, New York," 1947.

[2] "IEEE Standard for Wireless LAN MEdium Access Control (MAC) and Physical Layer (PHY) Specifications, P802.11."

[3] Y.-C. Hu, A. Perrig, and D. B. Johnson, "Ariadne: A Secure on-demand routing protocol for ad hoc networks," In Proc. of MobiCom '02, pp. 12-23, 2002.

[4] C. Perkins, E. Belding-Royer, and S. Das, "Ad hoc Ondemand Distance Vector (AODV) routing," IETF RFC 3561, 2003.

[5] J. Broch, D. B. Johnson, and D. A. Maltz, "The dynamic source routing protocol for mobile ad hoc networks," Internet-Draft Version 03, IETF, 1999.

[6] T. Clausen and P. Jacquet, "Optimized link state routing protocol (OLSR)," Tech. Report RFC3626, IETF, 2003.

[7] L. Zhou and Z. Haas, "Securing ad hoc networks," IEEE Network Magazine, vol. 13, 1999

[8] Y. Zhang and W. Lee, "Intrusion detection in wireless adhoc networks," In Proc. of MobiCom '00, pp. 275-283, 2000.

[9] J. P. Hubaux, L. Buttyan, and S. Capkun, "The quest for security in mobile ad hoc networks," In Proc. of ACM MobiHoc '01, pp. 146-155, 2001.

[10] P.-W. Yau and C. J. Mitchell, "Security vulnerabilities in ad hoc networks," In Proc. of the 7th Int. Symposium on Communication Theory and Applications, pp. 99-104, 2003.
[11] Y.-C. Hu, D. B. Johnson, and A. Perrig, "SEAD: secure efficient distance vector routing for mobile wireless adhoc networks," In Proc. of the IEEE WMCSA '02, 2002.

[12] P. Papadimitratos and Z. Haas, "Secure routing for mobile ad hoc networks," In Proc. of CNDS '02, pp. 27-31, 2002.

[13] L. Buttyan and J.-P. Hubaux, "Enforcing Service Availability in Mobile Ad-hoc WANS," In Proc. of MobiHOC' $00,2000$.

[14] S. Zhong, Y. Yang, and J. Chen., "Sprite: A simple, cheatproof, credit-based system for mobile ad hoc networks," In Proc. of Infocom '03, 2003.

[15] K. Balakrishnan, Deng, and P. Varshney, "TWOACK: Preventing Selfishness in Mobile Ad Hoc Networks," In Proc. of IEEE WCNC'05, pp. 2137-2142, 2005.

[16] S. Buchegger and J.-Y. L. Boudec, "The Effect of Rumor Spreading in Reputation Systems for Mobile Ad-hoc Networks," In Proc. of WiOpt '03, 2003.

[17] S. Buchegger and J. Y. LeBoudec, "Performance analysis of the CONFIDANT protocol: cooperation of nodes fairness in dynamic ad hoc networks," In Proc. of the ACM MobiHoc '02, pp. 226-236, 2002.

[18] S. Buchegger, C. Tissieres, and J. Y. LeBoudec, "A TestBed for Misbehavior Detection in Mobile Ad-hoc Networks - How Much Can Watchdogs Really Do?," In Proc. of the IEEE WMCSA '04, pp. 102-111, 2004.

[19] M. Conti, E. Gregori, and G. Maselli, "Towards Reliable Forwarding for Ad Hoc Networks," In Proc. of PWC '03, pp. 790-804, 2003.

[20] Q. He, D. Wu, and P. Khosla, "SORI: A secure and objective reputation-based incentive scheme for ad hoc networks," In Proc. of IEEE WCNC '04, pp. 825-830, 2004.

[21] J. Liu and V. Issarny, "Enhanced reputation mechanism for mobile ad hoc networks," In Proc. of the 2nd Int. Conf. on Trust Management, 2004.

[22] S. Marti, T. J. Giuli, K. Lai, and M. Baker, "Mitigating routing misbehavior in mobile ad hoc networks," In Proc. of ACM MobiCom '02, pp. 255-265, 2000.

[23] M. T. Refaei, V. Srivastava, L. DaSilva, and M. Eltoweissy, "A Reputation-based mechanism for Isolating Selfish Nodes in Ad Hoc networks," In Proc. of IEEE Mobiquitous '05, pp. 3-11, 2005.

[24] P. Michiardi and R. Molva, "CORE: A collaborative reputation mechanism to enforce node cooperation in mobile ad hoc networks," Proc. of the 6th Conference on Communications and Multimedia Security, pp. 107-121, 2002.

[25] P.-W. Yau and C. J. Mitchell, "Reputation Methods for Routing Security for Mobile Ad Hoc Networks," In Proc. of SympoTIC '03, pp. 130-137, 2003.

[26] H. Yang, J. Shu, X. Meng, and S. Lu, "SCAN: SelfOrganized Network-Layer Security in Mobile Ad Hoc Networks," IEEE Jornal on Selected Areas in Communications, vol. Vol. 24, NO. 2, pp. 261-273, 2006.

[27] Y. Rong, S. K. Lee, and H.-A. Choi, "Detecting stations cheating on backoff rules in 802.11 networks using sequential analysis," In Proc. IEEE INFOCOM '06, 2006.

[28] S. R. J. S. Baras and I. Koutsopoulos, "A framework for MAC protocol misbehavior detection in wireless networks," In Proc. of the 4th ACM workshop on Wireless security, Cologne, Germany, pp. 33-42. 